\title{
CORRECTIONS TO MY PREVIOUS PAPER "TOPICS IN THE FUNCTIONAL CALCULUS"*
}

\author{
BY L. M. GRAVES
}

Professor Chittenden has called to my attention that on the first page of my paper I quoted an erroneous statement from his memoir On general topology $\cdots . \dagger$ This statement was to the effect that the set-function $H$ defined by him on page 298 is always additive, that is, $H(D+E)=H(D)+H(E)$ for every pair of sets $D$ and $E$. Chittenden has communicated to me the following example in which the desired property fails to hold. Let limiting relations on the axis of reals be defined as usual, except that the origin belongs to the derivative $K(E)$ of a set $E$ only in case it is both a limit point in the ordinary sense of the subset of $E$ to the left of itself, and also a limit point of the subset of $E$ to the right of itself. If the original set-function $K$ is defined in this way, then the related function $H$ is identical with $K$, and so fails to be additive.

However, if the set function $H$ is defined in terms of a setfunction $K$ which is additive, it is easily shown that $H$ will also be additive. This was the case on page 642 of my paper, where a second reference to the process of Chittenden occurs. Moreover, if $K$ is an arbitrary set-function, a related function $L$ may be defined which is monotone, and then the function $L_{a}$ defined by the equation

$$
L_{a}(E)=L(E)+\sum_{D}\lfloor L(E+D)-L(D)\rfloor
$$

is always additive. From this it follows that the error does not essentially affect the statements made in my paper.

A second error in the paper (this one is not plagiarized) occurs at the bottom of page 642 . The last seven lines on page 642 and the first three lines on page 643 should be deleted, as the three statements contained in these ten lines are all erroneous. The exact nature of the function $H(E)$ defined by Chittenden, when

* This Bulletin, vol. 41 (1935), p. 641.

$\dagger$ Transactions of this Society, vol. 31 (1929), p. 290. 
applied to the example discussed in these lines, is unknown. In fact it is not clear that there exist sets $E$ for which $H(E)$ is not vacuous. But it is obvious that $H(E)$ is always contained in $K(E)$, where $K$ is the set-function (assumed additive) in terms of which $H$ is defined.

In the example in question it is true that an "accessible" topology can be defined in terms of neighborhoods in such a way that the function $L x_{n}$ defined in terms of these neighborhoods is identical with the original function $L$, and so that the set of all continuous functions is dense on the whole space.

Institute for Advanced Study

\section{ON $(2,2)$ PLANAR CORRESPONDENCES}

BY L. H. CHAMBERS

1. Introduction. Most of the existing literature dealing with $(2,2)$ planar transformation is of the type given by the product of two harmonic homologies. By this I mean that the pairs of points of the plane $\pi$ (or $\pi^{\prime \prime}$ ) are in harmonic homology. Papers of this type were given by E. Amson, ${ }^{*} \mathrm{~T}$. Kubota, $\dagger$ and P. Visalli. $\ddagger$ Barraco $\S$ defined an involutorial $(2,2)$ transformation of the plane by means of an involution between the tangents to a conic from points of the plane.

In this paper I shall consider only periodic $(2,2)$ transformations of period two. The treatment in each case, except those involving the Bertini involution, will be analytic. A synthetic treatment of some of the cases has been given by Sharpe and Snyder.|I I shall use the following theorems proved in their paper.

A necessary and sufficient condition that the two images of a point $P$ describe distinct loci as $P$ moves on a curve $C$ is that $C$ touches the branch curve at every non-fundamental point they have in common.

* Erlangen Dissertations, vol. 130 (1903-04).

† Science Reports, Tôhoku, vol. 6 (1918), and vol. 14 (1925).

¥ Circolo Matematico di Palermo, Rendiconti, vol. 3 (1889), pp. 165.

\& Giornale di Matematiche, vols. 53-54 (1915-16).

\| Transactions of this Society, vol. 18 (1918), pp. 409. 